\title{
Clinical acute pancreatitis following anterograde single balloon enteroscopy
}

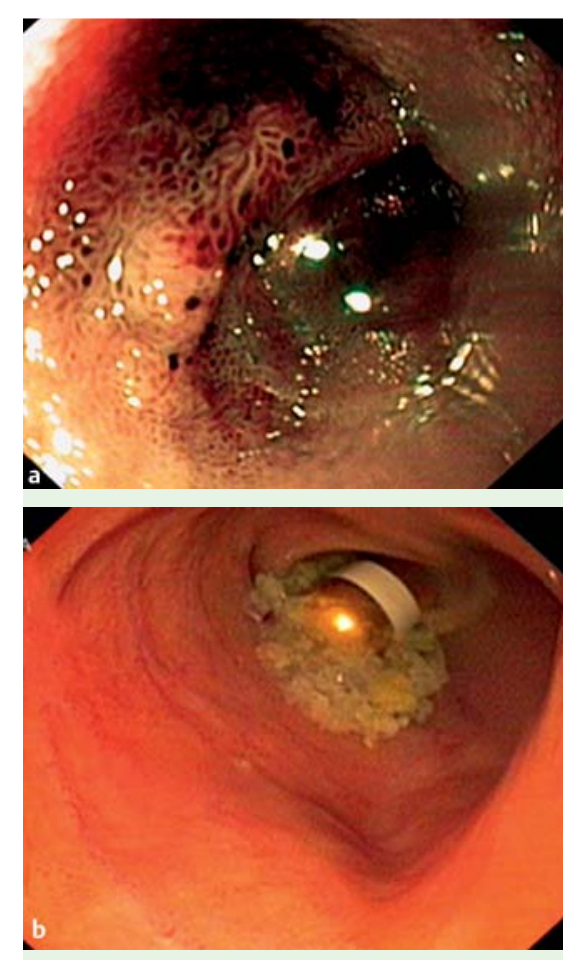

Fig. 1 a Single balloon enteroscopy (SBE) narrow band image showing ulcers in the small bowel. b SBE showing the retained capsule in the distal ileum.

A 37-year-old woman with suspicion of Crohn's disease underwent capsule endoscopy; however, the capsule was still in the small bowel even after 14 days. Anterograde single balloon enteroscopy (SBE) was planned to retrieve the capsule and to simultaneously carry out a biopsy. On SBE, multiple ulcers and one major stricture were found in the distal bowel with the capsule lying over the stricture ( Fig. 1 a,b). The capsule was successfully retrieved and multiple biopsy samples were taken, with the procedure lasting 3 hours. Following the procedure, the patient developed severe upper abdominal pain. Investigations revealed serum amylase of $1217 \mathrm{U} / \mathrm{L}$ at $24 \mathrm{~h}$ post procedure. Although previous computed tomography (CT) scans had shown a normal pancreas ( $\bullet$ Fig. 2), a further CT scan of the abdomen showed a bulky pancreas with peripancreatic fluid collections ( $\bullet$ Fig. 3). The patient was managed conservatively and discharged on day 7. The small bowel biopsy showed features consistent with Crohn's disease.

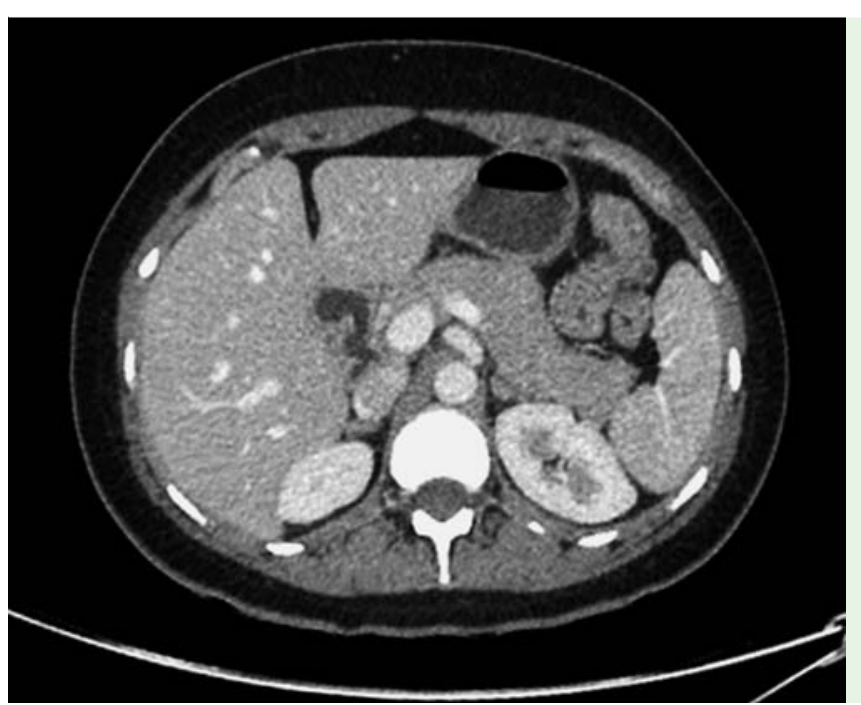

Fig. 2 Contrast-enhanced computed tomography (CT) scan taken 1 month before the single balloon enteroscopy showing a normal pancreas.

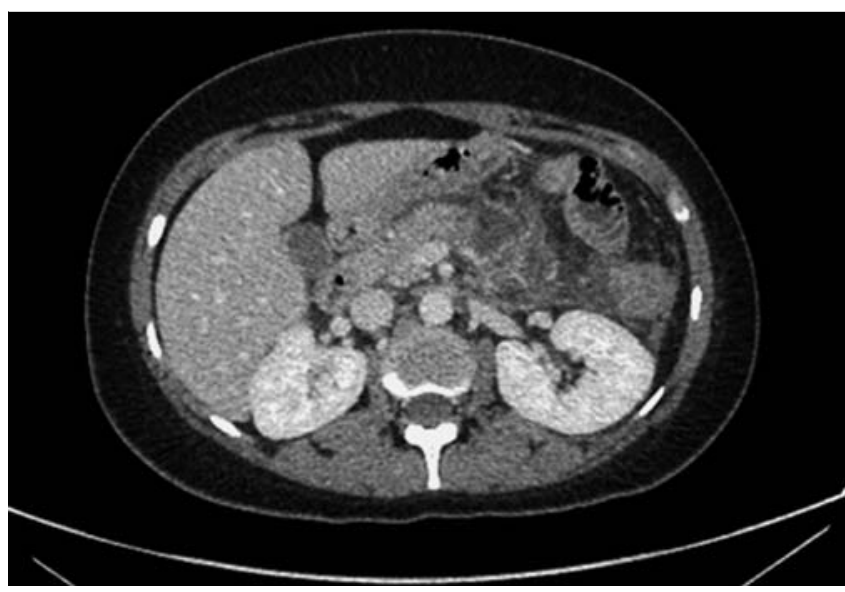

Fig. 3 Contrast-enhanced computed tomography (CT) scan taken at the time of pancreatitis showing a bulky body and tail of pancreas with ill-defined fluid collections in the peripancreatic region.

SBE is a safe endoscopic technique for the evaluation of the small bowel. Asymptomatic post-procedural hyperamylasemia is common after SBE, with one reported case of acute pancreatitis after retrograde SBE $[1,2]$. The present case is the first report of acute pancreatitis after anterograde SBE. Various theories have been postulated for hyperamylasemia after double/single balloon enteroscopy, such as repeated stretching of the small-bowel and/or mesenteric ligaments, prolonged mechanical stress on the pancreas due to repeated stretching by the endoscope, mechanical torsion of the pancreatic body during insertion of the endoscope, ischemia of the pancreas, direct trauma to the ampullary area, or a direct obstruction of the pancreatic duct by the insuf- flated balloon, but none has been universally accepted $[3,4]$.

Most studies on double/single balloon enteroscopy could not identify any risk factor for the associated abdominal pain, elevated pancreatic amylases, and acute pancreatitis [5]. Thus we advise close observation after a prolonged SBE procedure and that any patient with postprocedural abdominal pain should be evaluated for acute pancreatitis.

Endoscopy_UCTN_Code_CPL_1AI_2AD

Competing interests: None 
M. K. Sharma ${ }^{1}$, P. Sharma1, H. Garg1', L. Sehgal ${ }^{2}$, V. Bhatia ${ }^{1}$

1 Department of Hepatology, Institute of Liver and Biliary Sciences, New Delhi, India

2 Department of Anesthesia and Critical Care, Institute of Liver and Biliary

Sciences, New Delhi, India

\section{References}

1 Tsujikawa T, Saitoh Y, Andoh A et al. Novel single-balloon enteroscopy for diagnosis and treatment of the small intestine: preliminary experiences. Endoscopy 2008; 40: $11-15$

2 Kawamura T, Yasuda K, Tanaka K et al. Clinical evaluation of a newly developed singleballoon enteroscope. Gastrointest Endosc 2008; 68: 1112 - 1116

3 Aktas H, de Ridder L, Haringsma J et al. Complications of single-balloon enteroscopy: a prospective evaluation of 166 procedures. Endoscopy 2010; 42: 365-368

4 Groenen MJ, Moreels TG, Orlent $\mathrm{H}$ et al. Acute pancreatitis after double-balloon enteroscopy: an old pathogenetic theory revisited as a result of using a new endoscopic tool. Endoscopy 2006; 38: $82-85$

5 Pennazio M. Small-bowel endoscopy. Endoscopy 2008 ; 40: 835-842
Bibliography

DOI $10.1055 / \mathrm{s}-0030-1255892$

Endoscopy 2011; 43: E20 - E21

(c) Georg Thieme Verlag KG Stuttgart · New York . ISSN 0013-726X

\section{Corresponding author}

\section{Dr M. K. Sharma}

Institute of Liver and Biliary Sciences D1 Vasant Kunj

New Delhi-70

manojkumardm@gmail.com 